\title{
Laparoscopic gastric plication and its effect on saccharide and lipid metabolism: a 12-month prospective study
}

\author{
Marek Bužga ${ }^{1}$, Pavol Holéczy ${ }^{2}$, Zdeněk Švagera ${ }^{3}$, Pavel Zonča ${ }^{2}$ \\ ${ }^{1}$ Department of Physiology and Pathophysiology, University of Ostrava, Ostrava, Czech Republic \\ ${ }^{2}$ Department of Surgical Studies, Faculty of Medicine, University of Ostrava, Ostrava, Czech Republic \\ ${ }^{3}$ Department of Biomedical Sciences, Faculty of Medicine, University of Ostrava, Czech Republic
}

Videosurgery Miniinv 2015; 10 (3): 398-405

DOI: $10.5114 /$ wiitm.2015.54103

\begin{abstract}
Introduction: Laparoscopic greater curvature plication (LGCP) is a novel restrictive technique that reduces gastric volume by plication of the greater curvature. The advantage of LGCP is its reversibility in comparison to laparoscopic sleeve gastrectomy. Nowadays, the long-term LGCP efficacy, safety and metabolic effect are being investigated.

Aim: To assess body composition, clinical complications and metabolic changes in obese patients 6 and 12 months after laparoscopic greater curvature plication.

Material and methods: A total of 70 subjects underwent LGCP; 52 of them (33 women and 19 men) completed 1-year follow-up study. Anthropometry and biochemical parameters (glucose, glycated haemoglobin, lipids, ghrelin, leptin, adiponectin and fibroblast growth factor 21 [FGF-21]) were assessed before and 3, 6, and 12 months after surgery.

Results: All study participants exhibited statistically significant weight loss at both 6 and 12 months following the LGCP compared to baseline, with significant reductions in body composition - body weight, body mass index, percentage excess weight loss (\%EWL), and percentage excess BMI loss (\%EBL) ( $p \leq 0.001)$. Moreover, significant lowering of glucose and glycated haemoglobin, triacylglycerols and leptin was observed 12 months after LGCP. On the other hand, plasma concentrations of ghrelin, adiponectin and LDL cholesterol increased significantly. Total cholesterol, LDL cholesterol and FGF-21 levels did not change significantly.

Conclusions: Laparoscopic greater curvature plication appears to be a procedure with good restriction results, which might be mediated through alteration in incretin metabolism. Technical aspects and standardization of the procedure still remain to be worked out.
\end{abstract}

Key words: weight loss, ghrelin, fibroblast growth factor 21, metabolic effect, gastric plication.

\section{Introduction}

Over the past two decades, obesity has become a serious global health problem. The International Association for the Study of Obesity/International Obesity Task Force, a WHO body, has reported that approximately 1 billion adults worldwide are overweight and another 475 million adults are obese [1]. In the countries of Central and Eastern Europe, including the Czech Republic, the prevalence of obesity stands as a forefront issue in all epidemiological studies. Results of the latest large epidemiological study conducted in the Czech Republic reveal that $30 \%$ of Czechs are overweight and $25 \%$ of them suffer from obesity [2].

There are several therapeutic approaches to obesity. In most cases, the first choice is lifestyle change focused on balanced dietary intake and physical ac-

\section{Address for correspondence}

Zdeněk Švagera MSc, PhD, Department of Biomedical Sciences, Faculty of Medicine, University of Ostrava, Syllabova 19, Ostrava,

Czech Republic, phone: +42 0597374 121, e-mail: zdenek.svagera@osu.cz, zdenek.svagera@fno.cz 
tivity. Pharmaceutical therapy, the principal aims of which are to support change in dietary habits and prevent decrease in basal metabolism, is another option. However, since the end of the 1990s, surgical treatment (bariatric/metabolic surgery) has proved to be the most effective obesity treatment $[3,4]$. Unlike conservative treatment, which fails in more than $80 \%$ of patients in the long term, bariatric surgery results in long-term success in more than $80 \%$ of patients [5]. It is the only treatment that produces long-term weight loss in morbidly obese patients [6].

Like all surgical procedures, bariatric surgery requires appropriate training [7] and carries its own risks [8]. Although cure of associated co-morbidities cannot be guaranteed, more than $75 \%$ of obese patients experience complete or partial post-operative remission of most obesity-associated health conditions (e.g type 2 diabetes, dyslipidaemia, and hypertension) [9].

Surgical treatment for obesity is either malabsorptive, or restrictive, or a combination of both. Laparoscopic adjustable gastric banding (LAGB) and laparoscopic sleeve gastrectomy (LSG) are the most popular restrictive procedures for morbid obesity. On the basis of a recent update of the Cochrane Database Systematic Review of 20 randomized controlled trials, LSG is superior to LAGB in terms of weight loss and comparable to the outcome of gastric bypass [10]. Laparoscopic greater curvature plication (LGCP) is a novel restrictive technique that reduces gastric volume by plication of the greater curvature. The mechanism of LGCP is notably similar to that of LSG in that both result in gastric tube formation and elimination of the greater curvature, but LGCP has the advantage of a reversible restrictive technique without the use of gastrectomy [11]. However, its long-term efficacy is under investigation, and there are very few studies that compare it with other bariatric procedures, including LSG.

\section{Aim}

The main purpose of this study was to assess body composition, clinical complications and metabolic changes in obese patients 6 and 12 months after laparoscopic greater curvature plication.

\section{Material and methods}

\section{Patients}

A total of 70 subjects aged 24 to 68 years underwent LGCP. The study was approved by the
Ethics Committee of the Faculty of Medicine, University of Ostrava, Czech Republic, in accordance with the ethical standards of the Helsinki Declaration of 1975, as revised in 2000. The subjects were patients of the Gastroenterology Care Centre, Department of Surgery of the Vitkovice Hospital, Czech Republic. Selection of subjects for the surgical treatment of obesity was carried out in accordance with the guidelines of the International Federation for the Surgery of Obesity and Metabolic Disorders (IFSO). The subjects had either body mass index $(\mathrm{BMI}) \geq 40 \mathrm{~kg} / \mathrm{m}^{2}, \mathrm{BMI} \geq 35 \mathrm{~kg} / \mathrm{m}^{2}$ with associated co-morbidities, or BMI $<35 \mathrm{~kg} / \mathrm{m}^{2}$ with a history of weight loss resulting from intensive therapy followed by regaining of weight. Patients with history of stomach surgery, gastric and duodenal ulcers, thyroid disorders, gastrointestinal disorders associated with intestinal resorption dysfunction and hypolipidaemic and anti-diabetic treatment were excluded.

Out of 70 enrolled subjects, 52 subjects (33 women and 19 men) completed the study. Eighteen subjects were rejected from the study due to loss of follow-up. All patients underwent pre-operative and follow-up examinations $(3,6$, and 12 months after surgery). The assessment procedures - anthropometry, monitoring of serum concentrations of fasting glucose and relevant hormones - were identical on each assessment occasion. This was a prospective, non-randomized, open-label, single-centre, observational safety and feasibility study.

\section{Surgical technique}

The operations were performed under general anaesthesia, in supine position, with legs apart. A laparoscopic approach with five ports was used. Two 5-mm ports and three $10-\mathrm{mm}$ ports were placed in the upper abdomen according to a standard schema. Dissection of the greater curvature started $4 \mathrm{~cm}$ proximally from the pylorus along the whole greater curvature. Two proximal short gastric veins were preserved. Afterwards, the greater curvature was plicated with one or two rows of continuous non-absorbable monofilament suture (18 vs. 34 patients, respectively). The first three procedures were carried out under endoscopic observation (Photo 1); thereafter the procedures were performed without endoscopic guidance or bougie. The anatomic landmarks were free margins of short gastric veins. Tilting of the operating table was used to obtain better vision 


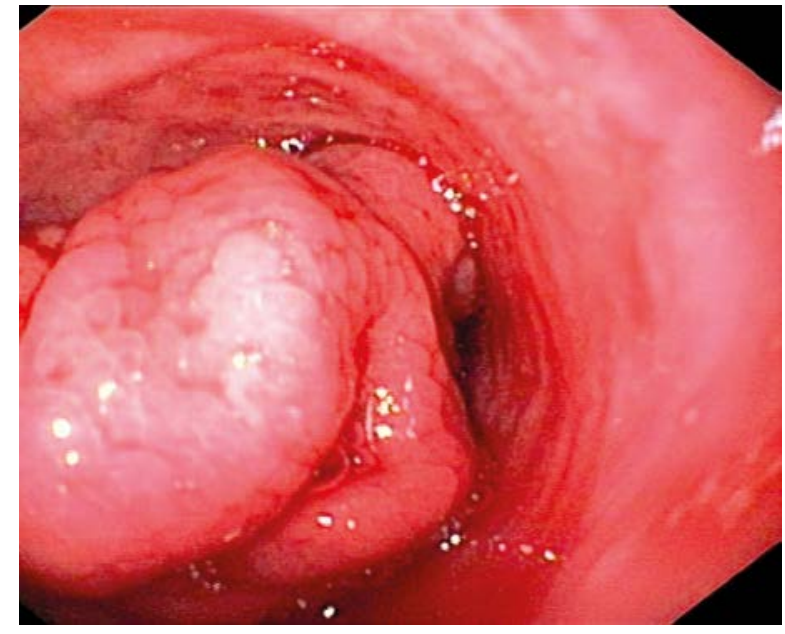

Photo 1. LGCP - endoscopic view

in the operating field. No antibiotic prophylaxis was used. At the end of the procedures, serotonin 5-HT3 receptor antagonist was administered to prevent nausea and vomiting. Proton pump inhibitor was administered before the operation, $20 \mathrm{mg}$ orally and also intravenously after the operation until the resolution of nausea.

\section{Post-operative care}

Following the operation, the patients were transferred to the ICU, usually for $24 \mathrm{~h}$. Oral liquids were allowed depending on tolerance from $4 \mathrm{~h}$ post-operatively. Early mobilization was forced mostly $6 \mathrm{~h}$ after the operation. The special "bariatric diet" called BS 1 to 7, was administered in the next days. Patients were discharged only when they were able to eat a BS 7 diet (solid food).

\section{Anthropometric assessment}

A basic anthropometric examination was carried out, including measurements of body weight, height, waist and hip circumference, and calculation of BMI. Body composition was determined by dual-energy $X$-ray absorptiometry (DXA, Hologic Discovery A, Waltham, USA).

\section{Weight loss}

The main study objective was to assess the weight loss after LGCP. The weight loss assessments included absolute weight loss (AWL), percentage of total weight loss (\%TWL), percentage of excess weight loss (\%EWL), and changes in BMI. Percentage of EWL was calculated using ideal body weight according to the middle of the 1983 Metropolitan Life Insurance tables for median frame. The weight was measured by electronic scales on the day of surgery and at 3, 6, and 12 months after surgery.

\section{Biochemical assessment}

Venepuncture was performed in the morning after overnight fasting a week before the planned procedure, and 3, 6 and 12 months after it. Blood samples, except the sample for glycated haemoglobin determination, were centrifuged at $2500 \mathrm{~g}$ for $6 \mathrm{~min}$ at $4^{\circ} \mathrm{C}$. Serum concentrations of the following substances were assayed: glucose, triacylglycerols (TG), total cholesterol (TC), high-density lipoprotein (HDL) and low-density lipoprotein (LDL) cholesterol (AU 5420, Beckman Coulter, Inc., USA). To reduce analytical variation of hormones and cytokines of fat tissue, the serum samples were aliquoted into two vials ( $2 \mathrm{ml}$ each) and stored at $-80^{\circ} \mathrm{C}$ until the time of analysis. Serum levels of leptin, adiponectin and ghrelin were analysed by the ELISA method using the sandwich sets (Biovendor - Laboratorni medicina, Czech Republic) by ELISA technology (DSX, Dynex Technologies, Chantilly, VA, USA). Fibroblast growth factor 21 (FGF21) was determined by multiplex assay developed by Biovendor - Laboratorni medicina using barcoded magnetic beads, and performed on Biocode-100A (Applied Biocode, USA). Glycated haemoglobin $\left(\mathrm{HbA}_{1 \mathrm{c}}\right)$ was measured by HPLC (Tosoh G8, Tosoh Bioscience Inc., CA, USA).

\section{Statistical analysis}

The anthropometry and body composition measurement results were evaluated by Student paired $t$-test. F-test based on a linear mixed model was used to compare concentrations of biochemical parameters pre- and post-operatively. Surgical complications were evaluated by a $\chi^{2}$ test and two-sample Wilcoxon rank-sum (Mann-Whitney) test. The level of significance was set at 0.05 . These statistical data were processed with the Stata v.10 program and statistical software $R$ version 2.14.1. (R Development Core Team 2013).

\section{Results}

A total of 70 patients (51 women and 29 men) underwent surgery from January 2011 to Decem- 
ber 2013. A total of 18 patients dropped out of the study. Fifty-two patients (33 women and 19 men) finished the study. Their initial mean age and mean weight were 42.1 years and $124.4 \mathrm{~kg}$, respectively. The mean BMI was $43.3 \mathrm{~kg} / \mathrm{m}^{2}$, including 3 patients with a $\mathrm{BMI}$ of $>50 \mathrm{~kg} / \mathrm{m}^{2}$. The mean duration of the procedure was $58.1 \mathrm{~min}$ (ranging from 45 to $90 \mathrm{~min}$ in one-row suture) versus 75 min (ranging from 50 min to 105 min in two-row suture) - a highly significant difference. The length of hospital stay did not differ significantly between one- and two-row plication.

Table I presents the weight loss outcomes and body composition in our cohort study, including the significant reductions noted in body fat mass and in lean body mass. The patients achieved a mean \%EWL of 51.5 after 12 months post-operatively, and a mean \%EBL of 56.9 during this period. All study participants exhibited statistically significant weight loss both at 6 and 12 months following the LGCP compared to baseline, with significant reductions in body composition - body weight, BMI, \%EWL, and $\% \operatorname{EBL}(p \leq 0.001)$.

\section{Biochemical variables}

Serum concentrations of glucose, triacylglycerols, leptin and blood concentrations of glycated haemoglobin were significantly lower 12 months after LGCP. On the other hand, plasma concentrations of ghrelin and adiponectin increased significantly. Similar to LSG studies, HDL cholesterol levels increased and triacylglycerol levels decreased. Low-density lipoprotein cholesterol, total cholesterol and FGF-21 levels did not change significantly.
The changes in biochemical parameters are summarized in Table II.

\section{Complications}

All operations were performed laparoscopically without any need for conversion. Laparoscopic greater curvature plication were performed in 18 patients with one row of sutures. The remaining 34 patients received procedure with two rows of sutures. One death was reported one year after the surgery without any connection with the operation. We compared the two subgroups (one-row plication to two-row plication) regarding surgical complications. There were 16 (88.9\%) patients without complications in the first subgroup versus 23 (67.6\%) patients in the second. In the one-row subgroup one repeated procedure was needed for partial release of the stomach wall plication or stomach dilatation and one gastro-gastric protrusion was recorded (both represent 5.6\%) (Photos 2, 3). The two-row subgroup required 5 repeated procedures for stomach dilatation or partial release (14.7\%); in 2 cases the plication was completely released after spontaneous partial release $(5.9 \%)$. The patients will undergo a sleeve gastrectomy later. Gastric wall perforation was observed in 1 (2.9\%) case. Partial release without further surgical intervention occurred in 2 (5.9\%) patients. An attempt to repair 1 partial release was technically impossible due to dense adhesions of stomach, spleen and liver. There were more complications observed in the two-row subgroup, but due to small numbers there were no statistically significant differences recorded $\left(\chi^{2}-p=0.177\right)$.

Table I. Anthropometric parameter changes 6 and 12 months after surgery ( $n=52,33$ women and 19 men)

\begin{tabular}{|lccc|}
\hline Parameter & Pre-operative examination & 6 months after surgery & 12 months after surgery \\
\hline Weight $[\mathrm{kg}]$ & $124.4 \pm 19.7$ & $107.2 \pm 18.2^{\mathrm{a}}$ & $102.0 \pm 17.8^{\mathrm{a}}$ \\
\hline Weight loss $[\mathrm{kg}]$ & - & $17.9 \pm 6.9^{\mathrm{a}}$ & $28.5 \pm 15.5^{\mathrm{a}}$ \\
\hline BMI $\left[\mathrm{kg} / \mathrm{m}^{2}\right]$ & $43.3 \pm 10.6$ & $36.8 \pm 10.4^{\mathrm{a}}$ & $33.3 \pm 9.4^{\mathrm{a}}$ \\
\hline EWL $(\%)$ & - & $41.7 \pm 7.3$ & $51.5 \pm 12.7^{\mathrm{a}}$ \\
\hline EBL $(\%)$ & - & $45.8 \pm 14.7$ & $56.9 \pm 24.3^{\mathrm{a}}$ \\
\hline Fat DXA $[\mathrm{kg}]$ & $55.9 \pm 10.6$ & $41.6 \pm 10.2^{\mathrm{a}}$ & $37.8 \pm 13.1^{\mathrm{a}}$ \\
\hline Fat DXA $(\%)$ & $45.1 \pm 5.7$ & $40.1 \pm 6.4^{\mathrm{a}}$ & $37.2 \pm 7.5^{\mathrm{a}}$ \\
\hline LBM DXA $[\mathrm{kg}]$ & $68.3 \pm 12.6$ & $62.1 \pm 12.2^{\mathrm{b}}$ & $62.7 \pm 12.3^{\mathrm{Ns}}$ \\
\hline
\end{tabular}

${ }^{a}$ Comparison with pre-operative examination $p<0.001,{ }^{b}$ Comparison with pre-operative examination $p<0.05,{ }^{N S}$ Comparison with pre-operative examination $p>0.05, E W L$ - excess weight loss, EBL - excess BMI loss, LBM - lean body mass. 
Table II. Changes in biochemical parameters pre-, 6 and 12 months after the surgery $(n=52,33$ women and 19 men)

\begin{tabular}{|c|c|c|c|}
\hline Parameter & Pre-operative examination & 6 months post-operative & 12 months post-operative \\
\hline Glucose $[\mathrm{mmol} / \mathrm{l}]$ & $5.7 \pm 1.0$ & $5.3 \pm 0.7^{\mathrm{a}}$ & $5.1 \pm 0.8^{\mathrm{a}}$ \\
\hline Glycated haemoglobin (\%) & $4.3 \pm 1.1$ & $3.9 \pm 0.5^{\mathrm{a}}$ & $3.7 \pm 0.4^{\mathrm{a}}$ \\
\hline Ghrelin [ng/l] & $127.5 \pm 96.9$ & $190.2 \pm 98.2^{b}$ & $201.8 \pm 101.1^{b}$ \\
\hline Leptin $[\mu \mathrm{g} / \mathrm{l}]$ & $53.6 \pm 14.9$ & $28.6 \pm 13.7^{\mathrm{a}}$ & $23.3 \pm 12.1^{a}$ \\
\hline Adiponectin [mg/l] & $16.3 \pm 7.8$ & $17.9 \pm 3.1^{\mathrm{NS}}$ & $25.8 \pm 11.0^{a}$ \\
\hline FGF $21[\mathrm{ng} / \mathrm{l}]$ & $112.2 \pm 117.9$ & $94.2 \pm 207.7^{\mathrm{NS}}$ & $77.5 \pm 103.1^{\mathrm{NS}}$ \\
\hline $\mathrm{TC}[\mathrm{mmol} / \mathrm{l}]$ & $4.9 \pm 1.2$ & $4.82 \pm 0.84^{\mathrm{NS}}$ & $4.9 \pm 0.8^{\mathrm{NS}}$ \\
\hline $\mathrm{TG}[\mathrm{mmol} / \mathrm{l}]$ & $2.4 \pm 1.6$ & $1.4 \pm 0.5^{a}$ & $1.1 \pm 0.8^{a}$ \\
\hline $\mathrm{HDL}[\mathrm{mmol} / \mathrm{l}]$ & $1.1 \pm 0.3$ & $1.3 \pm 0.3^{b}$ & $1.5 \pm 0.3^{a}$ \\
\hline LDL [mmol/l] & $3.2 \pm 0.9$ & $3.0 \pm 0.7^{\mathrm{NS}}$ & $3.0 \pm 0.3^{\mathrm{NS}}$ \\
\hline
\end{tabular}

${ }^{a}$ Comparison with pre-operative examination $p<0.001,{ }^{b}$ Comparison with pre-operative examination $p<0.01$, ${ }^{N S}$ non-significant, TC - total cholesterol, $T G$ - triacylglycerols, $H D L$ - high-density lipoprotein cholesterol, $L D L$-low-density lipoprotein cholesterol.

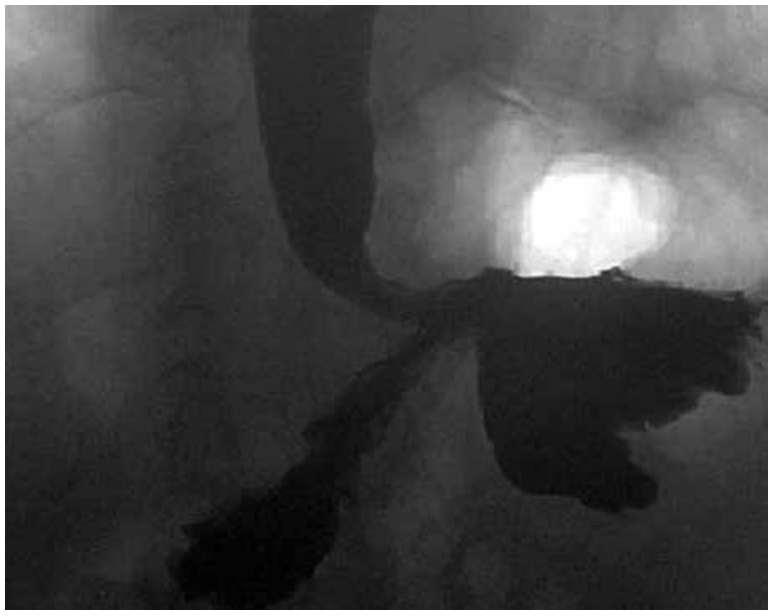

Photo 2. Gastro-gastric protrusion

\section{Discussion}

Weight loss results were similar to those of standard restriction procedures (LSG). Our LGCP weight loss results were comparable to other studies. Ramos et al. [12] reported a series of LGCP in 42 patients who achieved encouraging weight loss without major complications. The mean \%EWL was about $60 \%$ EWL at 12 months and $62 \%$ EWL at 18 months. Skrekas et al. [13] reported early-stage outcome in a series of 135 patients, showing that after a follow-up of 8-31 months (mean: 22.59 months), the mean \%EWL was 65.29. Shen et al. [11] reported a $58.8 \%$ EWL at 12 months in a series of 19 pa-

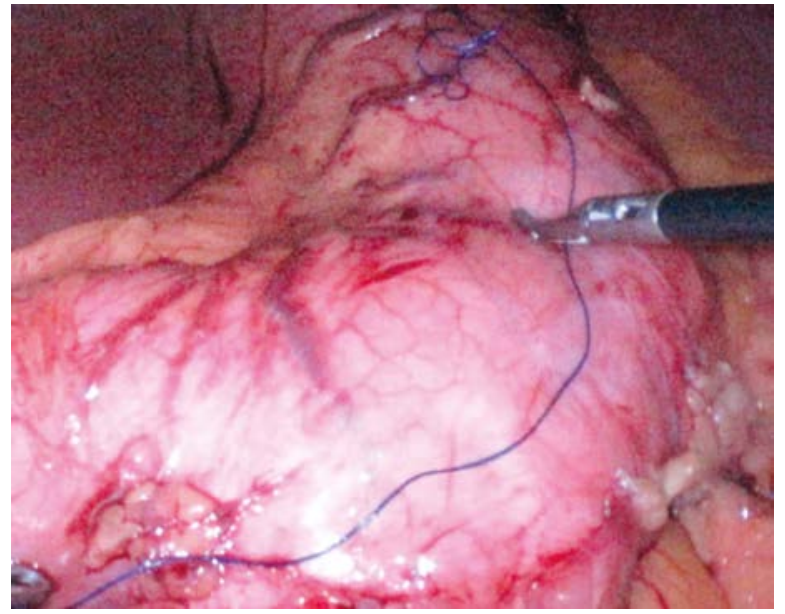

Photo 3. Spontaneous deplication

tients. A study combining LAGB with gastric plication conducted with 42 patients resulted in a remarkable final EWL of $62.6 \%$ [14]. These were, however, shortterm data from 6-month monitoring.

Data from recent studies indicate that LGCP is a method with very good general weight reduction and overweight reduction results. Nevertheless, it is necessary to point out that long-term data regarding evidence based medicine are lacking. So far, the global number of clinical studies is limited. A review of Taiwanese authors [15] presented interesting results. The systematic review covered 14 studies encompassing 1,450 LGCP patients. Mean pre-operative 
BMI ranged from $31.2 \mathrm{~kg} / \mathrm{m}^{2}$ to $44.5 \mathrm{~kg} / \mathrm{m}^{2}$, and $80.8 \%$ of the patients were female. The \%EWL for LGCP varied from $31.8 \%$ to $74.4 \%$ with follow-up from 6 months to 24 months. Our results are fully comparable in this regard, and can be considered as a successful weight loss. When LGCP and LSG methods are compared as to weight loss, EWL results are slightly lower after LGCP than LSG $[16,17]$. Yet the results do not differ very significantly. It is necessary to realize that overall weight loss due to any surgical method incomparably exceeds the weight loss that is achieved by conservative treatment itself [18].

Early reports with LGCP are promising, with a favourable short-term safety profile. However, it remains unclear if weight loss following LGCP is durable in the long term. Additional prospective comparative trials and long-term follow-up are needed to further define the role of LGCP in the surgical management of obesity [15].

\section{Metabolic effect of laparoscopic greater curvature plication}

Restrictive bariatric methods were considered as merely weight-reducing for a long time. Recently, studies began to emerge $[19,20]$, including meta-analyses [21], which have demonstrated a metabolic effect in both surgical and endoscopic restrictive procedures [22]. That is why LSG is no longer registered as an exclusively restrictive surgery, but as a so-called combination method [23]. The LGCP method, on the other hand, is a relative novelty, which has been introduced by a limited number of healthcare providers to date, even on a global scale. Metabolic effects of this method have practically not been studied.

So far, we have found a single reference to a study concerning metabolic outcomes of LGCP in the human organism [24]. This study noted a positive effect of LGCP on the postprandial changes of incretin pathways and hormones of the proximal GIT, as well as a positive effect on saccharide metabolism. Correspondingly, we found a significant decrease of glucose concentration and glycated haemoglobin in our study. Moreover, a significant decrease of total triacylglycerol levels and significant increase of HDL cholesterol levels were also observed in our cohort. Studies evaluating effects of LSG on the lipid spectrum have generated similar effects regarding serum lipids [16]. We consider the increase of total ghrelin levels, which were reported rising throughout the en- tire 12-month monitoring period, a significant finding. The Brazilian study by Ivano [25] provided similar results. It is known that ghrelin stimulates food intake during fasting because it leads to hunger, being an important hormone of body weight control [26]. But there are other hormones that regulate food intake and satiety, including YY peptide (PYY), glucagon like peptide (GLP-1) and leptin [27]. From this viewpoint, it is relatively difficult to evaluate the role of ghrelin as a single hormone influencing dietary intake. Leptin was another important factor monitored in our study. Leptin produced in white adipose tissue acts on the hypothalamus, leading to satiety and regulation of energetic balance. In our study, leptin showed a significant decrease 12 months after surgery, which is related to a decrease in the amount of adipose tissue after gastric plication. The decline in plasma leptin levels was similarly reported in bariatric surgery studies.

We found a mild, but not significant, decrease in FGF21 levels 12 months following the surgery. FGF21 has been considered a metabolic hormone regulated by nutritional status, with beneficial effects on glucose homeostasis and lipid metabolism in animal models [28]. In humans, increased FGF21 levels are associated with obesity in both children [29] and adults [30], indicating a connection between FGF21 and body fat mass. Studies analysing the response of FGF21 to weight loss in humans had controversial findings. Mai et al. [31] found that moderate weight loss ( $~ 5 \mathrm{~kg})$ did not induce changes of FGF21 levels in 30 obese subjects following a hypocaloric diet and physical activity regimen for 6 months. The same results were found in 23 non-diabetic, morbidly obese subjects 1 year after laparoscopic Roux-en-Y gastric bypass and laparoscopic sleeve gastrectomy [32]. However, a recently published work describes significant decreases in levels of FGF21 in 17 obese females undergoing laparoscopic sleeve gastrectomy [33], which is in accordance with our results.

\section{Conclusions}

Considering all results, LGCP appears to be a procedure with good restriction results, which might be mediated through alteration in incretin metabolism. Technical aspects and standardization of the procedure still remain to be worked out.

Bearing in mind the limitations of our study, such as the relatively small size of our cohort, the lack 
of a controlled comparison group and the relatively short duration of follow-up, our study suggests that LGCP can offer an effective addition to the arsenal of bariatric surgery with effects that may extend beyond gastric volume restriction. Larger and long-term studies are required to further explore the spectrum of metabolic/hormonal effects of LGCP and establish the role of this bariatric technique in the treatment of obesity.

\section{Acknowledgments}

This study was supported by a grant from the Ministry of Education of the Czech Republic, allocated via the University of Ostrava under registration number SGS06/LF/2014.

\section{Conflict of interest}

The authors declare no conflict of interest.

\section{References}

1. Walpole SC, Prieto-Merino D, Edwards P, et al. The weight of nations: an estimation of adult human biomass. BMC Public Health 2012; 12: 439.

2. Matoulek M, Svačina Š, Lajka J. The incidence of obesity and its complications in the Czech Republic. Vnitrni Lekarstvi 2010; 56: 1019-27.

3. Dadan J, Iwacewicz P, Hady HR. New approaches in bariatric surgery. Videosurgery Miniinv 2008; 3: 66-70.

4. Stanowski E, Pasnik K. Bariatric surgery - the current state of knowledge. Videosurgery Miniinv 2008; 3: 71-86.

5. Fried M, Ribaric G, Buchwald JN, et al. Metabolic surgery for the treatment of type 2 diabetes in patients with BMI $<35 \mathrm{~kg} / \mathrm{m}(2)$ : an integrative review of early studies. Obes Surg 2010; 20: 776-90.

6. Olbers T, Bjorkman S, Lindroos A, et al. Body composition, dietary intake, and energy expenditure after laparoscopic Rouxen-y gastric bypass and laparoscopic vertical banded gastroplasty - a randomized clinical trial. Ann Surg 2006; 244: 715-22.

7. Buzink S, Soltes M, Radonak J, et al. Laparoscopic surgical skills programme: preliminary evaluation of grade I level 1 courses by trainees. Videosurgery Miniinv 2012; 7: 188-92.

8. Soltes M, Radonak J. A risk score to predict the difficulty of elective laparoscopic cholecystectomy. Videosurgery Miniinv 2014; 9: 608-12.

9. Wylezol M, Pasnik K, Dabrowiecki S, et al. Polish recommendations for bariatric surgery. Videosurgery Miniinv 2009; 4: S5-8.

10. Picot J, Jones J, Colquitt JL, et al. Weight loss surgery for mild to moderate obesity: a systematic review and economic evaluation. Obes Surg 2012; 22: 1496-506.

11. Shen DJ, Ye H, Wang YD, et al. Comparison of short-term outcomes between laparoscopic greater curvature plication and laparoscopic sleeve gastrectomy. Surg Endosc Other Interv Techn 2013; 27: 2768-74.
12. Ramos A, Neto MG, Galvao M, et al. Laparoscopic greater curvature plication: initial results of an alternative restrictive bariatric procedure. Obes Surg 2010; 20: 913-8.

13. Skrekas G, Antiochos K, Stafyla VK. Laparoscopic gastric greater curvature plication: results and complications in a series of 135 patients. Obes Surg 2011; 21: 1657-63.

14. Lee WJ, Lee KT, Ser KH, et al. Laparoscopic adjustable gastric banding (LAGB) with gastric plication: short-term results and comparison with LAGB alone and sleeve gastrectomy. Surg Obes Relat Dis 2015; 11: 125-30.

15. Ji Y, Wang YD, Zhu JH, et al. A systematic review of gastric plication for the treatment of obesity. Surg Obes Relat Dis 2014; 10: $1226-32$.

16. Buzga M, Holeczy P, Svagera Z, et al. Effects of sleeve gastrectomy on parameters of lipid and glucose metabolism in obese women -6 months after operation. Videosurgery Miniinv 2013; 8: 22-8.

17. Hady HR, Dadan J, Golaszewski P, et al. Impact of laparoscopic sleeve gastrectomy on body mass index, ghrelin, insulin and lipid levels in 100 obese patients. Videosurgery Miniinv 2012; 7: 251-9.

18. Buchwald H, Avidor Y, Braunwald E, et al. Bariatric surgery: a systematic review and meta-analysis. JAMA 2004; 292: 1724-37.

19. Abbatini F, Rizzello M, Casella G, et al. Long-term effects of laparoscopic sleeve gastrectomy, gastric bypass, and adjustable gastric banding on type 2 diabetes. Surg Endosc Other Interv Techn 2010; 24: 1005-10.

20. Lee WJ, Chong K, Ser KH, et al. Gastric bypass vs sleeve gastrectomy for type 2 diabetes mellitus a randomized controlled trial. Arch Surg 2011; 146: 143-8.

21. Buchwald H, Estok R, Fahrbach K, et al. Weight and type 2 diabetes after bariatric surgery: systematic review and meta-analysis. Am J Med 2009; 122: 248-81.

22. Buzga M, Machytka E, Klvana P, et al. Effects of the intragastric balloon MedSil (R) on weight loss, fat tissue, lipid metabolism, and hormones involved in energy balance. Obes Surg 2014; 24 : 909-15.

23. Fried M, Yumuk V, Oppert JM, et al. Interdisciplinary European Guidelines on metabolic and bariatric surgery. Obes Surg 2014; 24: 42-55.

24. Bradnova O, Kyrou I, Hainer V, et al. Laparoscopic greater curvature plication in morbidly obese women with type 2 diabetes: effects on glucose homeostasis, postprandial triglyceridemia and selected gut hormones. Obes Surg 2014; 24: 718-26.

25. Ivano F, Menacho A, Silva L, et al. Comparison of ghrelin plasma levels between pre and post operatory period in patients submitted to gastric plication associated with fundoplication. Obes Surg 2013; 23: 1064-5.

26. Gale SM, Castracane VD, Mantzoros CS. Energy homeostasis, obesity and eating disorders: recent advances in endocrinology. J Nutr 2004; 134: 295-8.

27. Kini S, Herron DM, Yanagisawa RT. Bariatric surgery for morbid obesity - a cure for metabolic syndrome? Med Clin North Am 2007; 91: 1255-71.

28. Iglesias P, Selgas R, Romero S, et al. Biological role, clinical significance, and therapeutic possibilities of the recently discovered metabolic hormone fibroblastic growth factor 21. Eur J Endocrinol 2012; 167: 301-9. 
29. Reinehr T, Woelfle J, Wunsch R, et al. Fibroblast growth factor 21 (FGF-21) and its relation to obesity, metabolic syndrome, and nonalcoholic fatty liver in children: a longitudinal analysis. J Clin Endocrinol Metabol 2012; 97: 2143-50.

30. Mraz M, Bartlova M, Lacinova Z, et al. Serum concentrations and tissue expression of a novel endocrine regulator fibroblast growth factor-21 in patients with type 2 diabetes and obesity. Clin Endocrinol 2009; 71: 369-75.

31. Mai K, Schwarz F, Bobbert T, et al. Relation between fibroblast growth factor-21, adiposity, metabolism, and weight reduction. Metabol Clin Exp 2011; 60: 306-11.

32. Woelnerhanssen B, Peterli R, Steinert RE, et al. Effects of postbariatric surgery weight loss on adipokines and metabolic parameters: comparison of laparoscopic Roux-en-Y gastric bypass and laparoscopic sleeve gastrectomy - a prospective randomized trial. Surg Obes Relat Dis 2011; 7: 561-8.

33. Haluzikova D, Lacinova Z, Kavalkova P, et al. Laparoscopic sleeve gastrectomy differentially affects serum concentrations of FGF19 and FGF-21 in morbidly obese subjects. Obesity 2013; 21 : $1335-42$.

Received: 22.07.2015, accepted: 19.08.2015 